\title{
Noninteracting PID Control using Precompensators with Series - Parallel Connections
}

\author{
Hee-Hyol Lee* \\ Fukuoka Inst. of Tech. \\ Won-Kyu Choi \\ CRESYS Co.,Ltd. \\ Michio Miyazaki \\ Karito Gakuin Univ. \\ Kageo Akizuki \\ Waseda Univ. \\ *E-mail:lee@ffit.ac.jp, 3-30-1 Wajirohigashi, Higashi-ku, Fukuoka 811-0295 JAPAN
}

\begin{abstract}
Controlled variables in multivariable systems interact each other in general, therefore, it is necessary to remove their interaction sufficiently for the design of control systems. A cross controller was used for removing the interaction thus far mainly, however, it was complicated and the controller was high-order. On the other hand, the precompensator by the inverse Nyquist array method was also used for noninteraction of the controlled variables.

However it is difficult to remove the interaction of the controlled system, which has very strong interaction, when the precompensator is used only one. This paper proposes the series and/or parallel connections of the precompensators for noninteracting PID control.
\end{abstract}

\section{Introduction}

In multivariable control systems, controlled variables interact each other in general. In this case one compensation for a control loop exerts harmful influence upon other control loop through the interaction. If we could take away this interaction by some ways for a complete one to one corresponds between the manipulated variable and the controlled variable, then the design of control systems results in the individual compensation of the single loop control.

A cross controller was used for removing the interaction thus far mainly, but it was complicated and the controller was high-order. On the other hand, the precompensator by the inverse Nyquist array method was also used for noninteraction of the controlled variables. However it is difficult to remove the interaction of the controlled system, which has very strong interaction, when the precompensator is used only one.

This paper proposes the series and/or parallel connections of the precompensators for noninteracting PID control, and the precompensators are applied to the control of the fluid temperature and liquid level interacting plant to confirm the effectiveness.

\section{Noninteracting precompensators by pseudo diagonalization}

Let the precompensator $G_{c}$ be a square matrix of order $m$.

$$
G_{c}=\left[h_{i j}\right]
$$

Then the $i j$ th element of $G(s)=G_{p}(s) G_{c}$ is represented by

$$
\ell_{i j}\left(j \omega_{0}\right)=\sum_{k=1}^{m} g_{i k}\left(j \omega_{0}\right) h_{k j}
$$

at $s=j \omega_{\theta}$, where $g_{i k}(s)$ is the $i k$ th element of $G_{p}(s)$.

Here the elements of the $p$ th row-vector $h_{p}=\left(h_{l p}, h_{2 p}\right.$, ..., $\left.h_{m p}\right)^{T}$ of $G_{c}$ is selected as that the square-sum of the absolute value of the non-diagonal elements of the $p$ th row-vector $h_{p}$ of $G\left(j \omega_{0}\right)$,

$$
\sum_{i=1, i \neq p}^{m}\left|\ell_{i p}\left(j \omega_{0}\right)\right|^{2}
$$

is minimized under condition of the constraint,

$$
h_{l p}{ }^{2}+h_{2 p}{ }^{2}+\ldots+h_{m p}{ }^{2}=1
$$


For evaluation of interaction, a diagonal dominance is used in general. Let $\hat{G}(s)$ be the inverse matrix of a rational function matrix $G(s)$. Given the controlled system $G_{p}(s)$ and taking the pseudo diagonalization of the precompensator $G_{c}$, if $\hat{G}(s)=\left(G_{p}(s) G_{c}\right)^{-1}$ becomes the diagonal dominance, then the open loop transfer function matrix $Q(s)=G_{p}(s) G_{c} K(s)$ is considered to be the diagonal dominance for a diagonal matrix $K(s)$. Therefore every loop of the main controller $K(s)$ can design independently neglecting effects of the non-diagonal elements of $\hat{G}(s)$.

We can evaluate the diagonal dominance graphically. The diagonal dominance of the inverse matrix $\hat{Q}(s)=\left\{\hat{q}_{i j}(s)\right\}$ of the transfer function matrix $Q(s)$ on the imaginary axis is equivalent to that one side of the inequalities is valid for all $\omega$,

$$
\begin{aligned}
& \left|\hat{q}_{i i}(j \omega)\right|>d_{i}(j \omega) \equiv \sum_{j=1, j \neq i}^{m}\left|\hat{q}_{i j}(j \omega)\right|, i=1,2, \cdots, m \\
& \left|\widehat{q}_{i i}(j \omega)\right|>d_{i}^{\prime}(j \omega) \equiv \sum_{j=i, j \neq i}^{m}\left|\widehat{q}_{j i}(j \omega)\right|, i=1,2, \cdots, m
\end{aligned}
$$

The inequalities (5) and (6) mean that the circle with its center at $\hat{q}_{i i}(j \omega)$ and with a radius of $d_{i}(j \omega)$ does not include the origin. The set of vector loci is the inverse Nyquist array, and $d_{i}(j \omega)$ and $d_{i}{ }^{\prime}(j \omega)$ are Gershgorin band, then the Gershgorin band shows a degree of the interaction for the controlled system.

\section{Series-Parallel connections of}

\section{precompensators}

We are sometimes confronted with the difficult that noninteraction could not be achieved by one precompensator for the controlled system, which has very strong interaction. Here we consider the connections of precompensators: series, parallel and their combination.

\section{Series connections:}

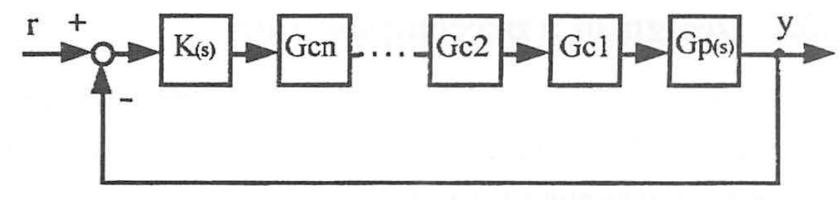

Figure 1: Series connections of precompensators
Parallel connections:

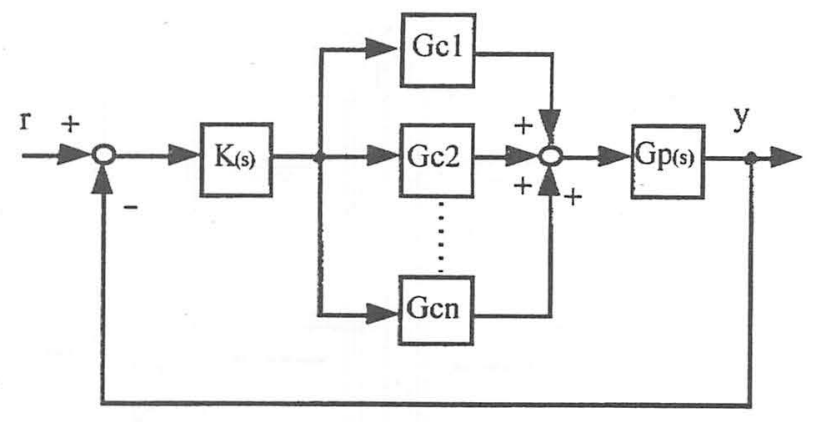

Figure 2 : Parallel connections of precompensators

For the series connections, the first precompensator $G_{c l}$ is designed using a typical hunting frequency $\omega_{0 /}$, and the diagonal dominance is evaluated by the inverse Nyquist array method. If the diagonal dominance does not succeed, the second precompensator $G_{c 2}$ is designed at $\omega_{02}$, which is the frequency of strong interaction, for the new controlled system $G_{p}(s)^{\prime}=G_{p}(s) G_{c l}$. The precompensators are connected one by one in this manner. On the hand, for the parallel connections, every precompensator $G_{c i}, i=1,2$, $\ldots, n$, is designed using the hunting frequencies $\omega_{0 i}$, and then they are connected in parallel.

\section{Noninteracting PID control using precompensators}

\subsection{Experimental plant}

The fluid temperature and liquid level experimental plant used in this study is composed of a warm water tank, a cold water tank and a mixed tank. Cold water and warm water are supplied to the mixed tank by two pumps, and the fluid temperature and the liquid level of the mixed tank are measured by a thermocouple and a level meter, respectively. This mixed tank is a closed tank with a constant flow valve, and the controlled system has very strong interaction between the fluid temperature and liquid level controlled variables. The construction of the experimental plant and the step responses of the plant are illustrated in Fig.3 and Fig.4, respectively, and the transfer functions deduced from the step responses are as follow

$$
G_{T}(s)=\frac{50277.498 s^{2}-2320.5 s+35.7}{484466.55 s^{3}+23768.33 s^{2}+409 s+1}
$$




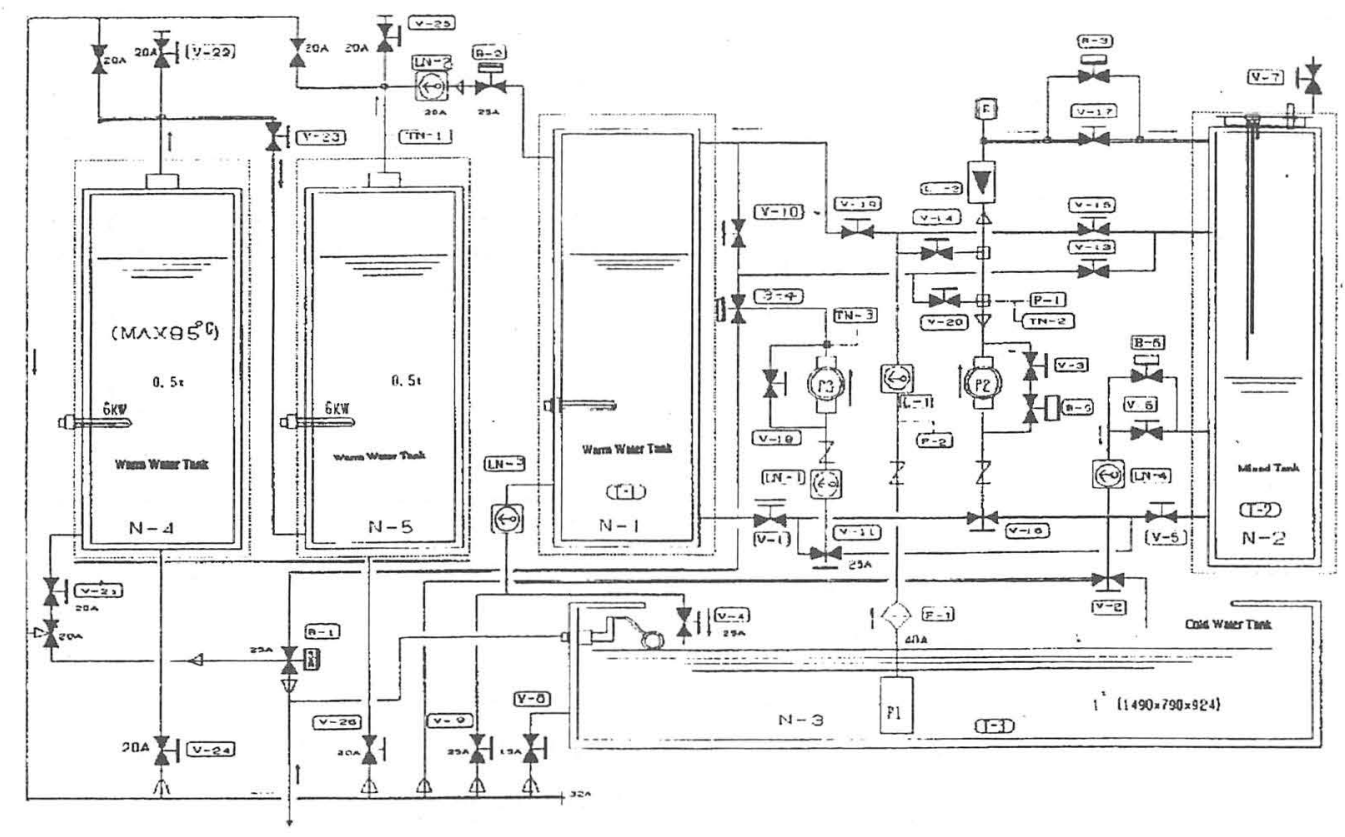

Figure 3 : Construction of the experimental plant
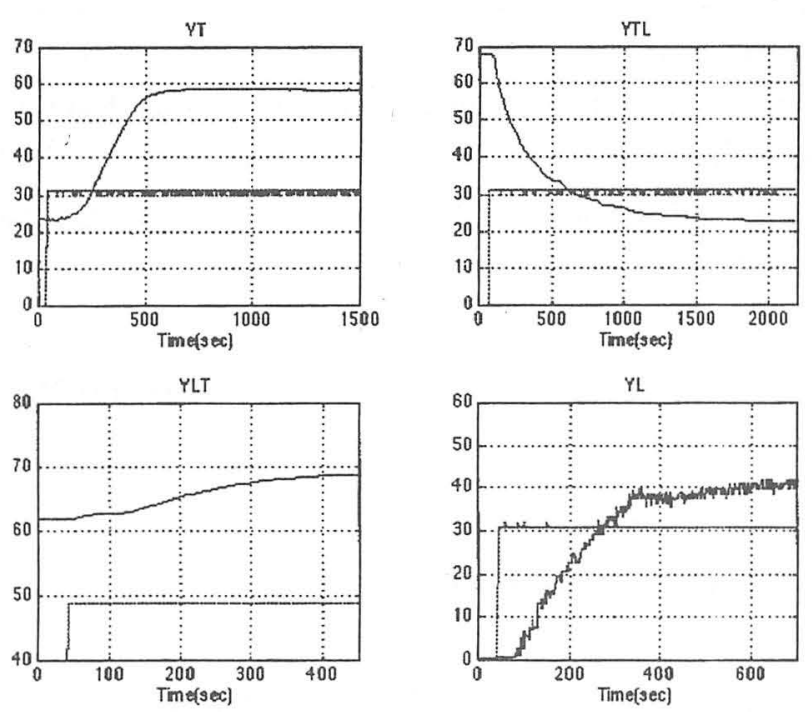

Figure 4 : Step responses of the plant

$$
\begin{aligned}
& G_{L T}(s)=\frac{76.893 s^{2}-80.94 s+28.4}{460.275 s^{3}+487.2075 s^{2}+172.85 s+1} \\
& G_{T L}(s)=\frac{1526.514 s^{2}-458 s+45.8}{10132.32 s^{3}+3037.33 s^{2}+314 s+1} \\
& G_{L}(s)=\frac{6376.32 s^{2}-885.6 s+41}{40124.16 s^{3}+5728.32 s^{2}+279.6 s+1}
\end{aligned}
$$

\subsection{PID control by each single loop}

For the controlled variables of the fluid temperature and liquid level, we design PID controllers for the each loop regarding other controlled variable as a disturbance.
Figure 5 is the result of the control, and it shows that the hunting occurs at two controlled variables with main period of about 4.3 [min].

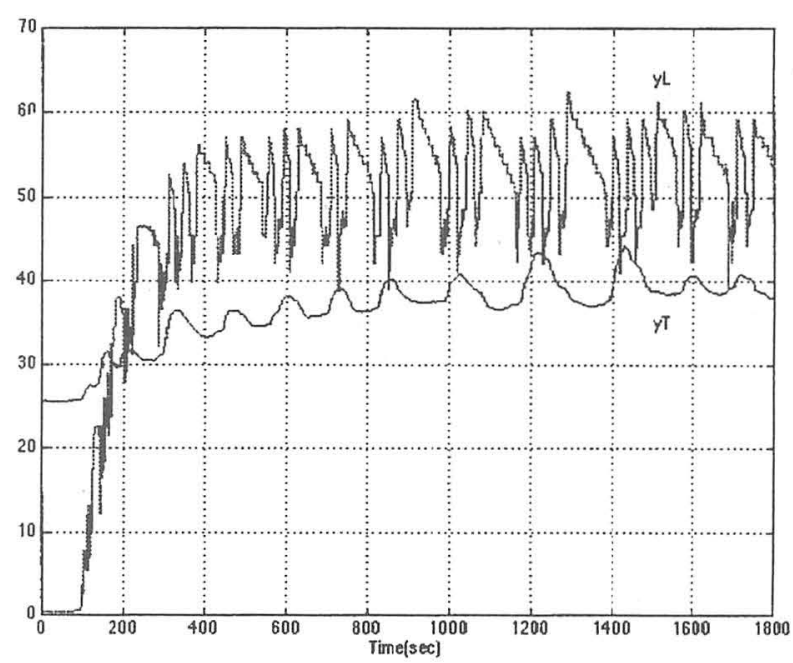

Figure 5: Result of the PID control

\subsection{Series-Parallel connection of precompensators}

\subsubsection{Design of a precompensator}

For the transfer function Eq.(7) of the fluid temperature and liquid level interacting plant, the precompensator $G_{c l}$ is designed at the hunting frequency $\omega_{0 l}=0.0245$ as

$$
G_{c 1}=\left[\begin{array}{rr}
0.6845 & 0.9597 \\
-0.7290 & 0.2812
\end{array}\right]
$$


The inverse Nyquist diagrams and Gershgorin bands of the loop transfer function $G(s)=G_{p}(s) G_{c l}$ are illustrated in Fig.6, and the diagonal dominance does not succeed.
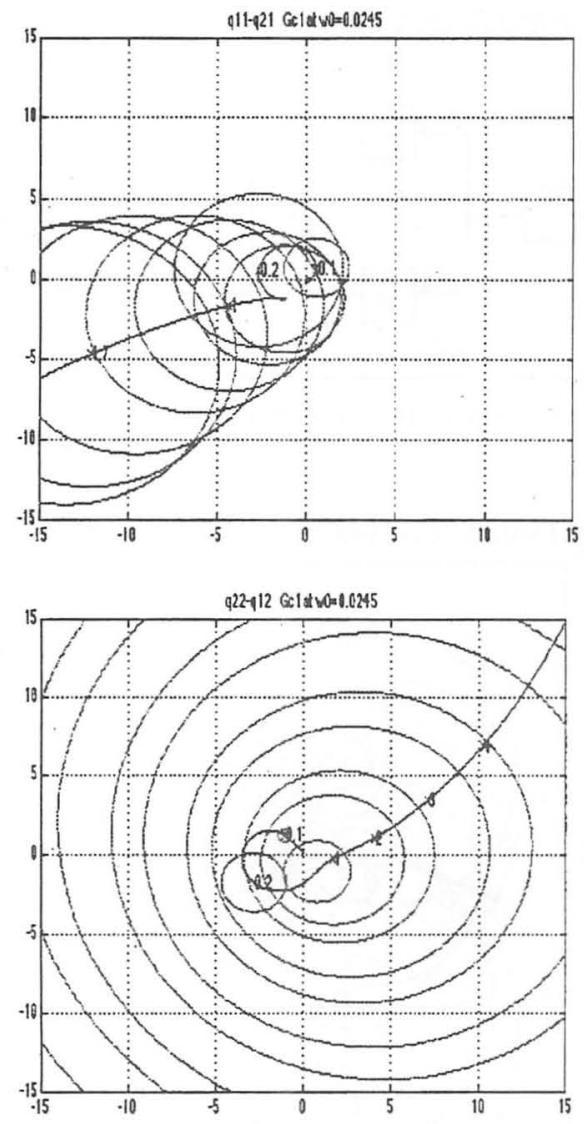

Figure 6: Inverse Nyquist diagram by $G_{c l}$

\subsubsection{Series connection of precompensators}

When we use one precompensator, it is difficult to realize the diagonal dominance for the controlled system with very strong interaction. Accordingly, we connect the precompensators one by one. A new precompensator $G_{c 2}$ is connected in a series, and designed at $\omega_{02}$, which is the frequency of strong interaction, regarding the transfer function $G_{p}(s) G_{c I}$ as a new controlled system $G_{p}(s)^{\prime}$. If the diagonal dominance does not realize, then a further precompensator $G_{c 3}$ is connected in a series and designed in same manner. The block diagram of the precompensators connected in a series to the third stage is shown in Fig.7, and then the precompensator $G_{c I} G_{c 2} G_{c 3}$ is as

$$
G_{c 1} G_{c 2} G_{c 3}=\left[\begin{array}{ll}
0.1858 & 0.2306 \\
0.1973 & 0.1544
\end{array}\right]
$$

The inverse Nyquist diagram and Gershgorin bands are shown in Fig.8, and then the diagonal dominance is realized at every frequency.

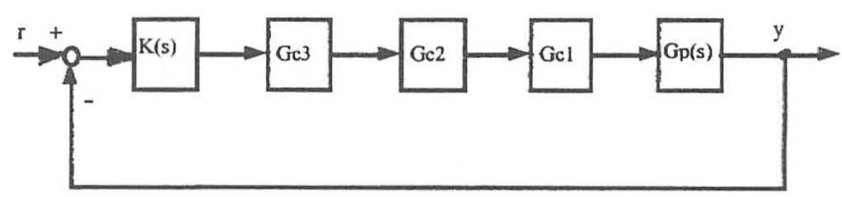

Figure 7 : Block diagram of series connection
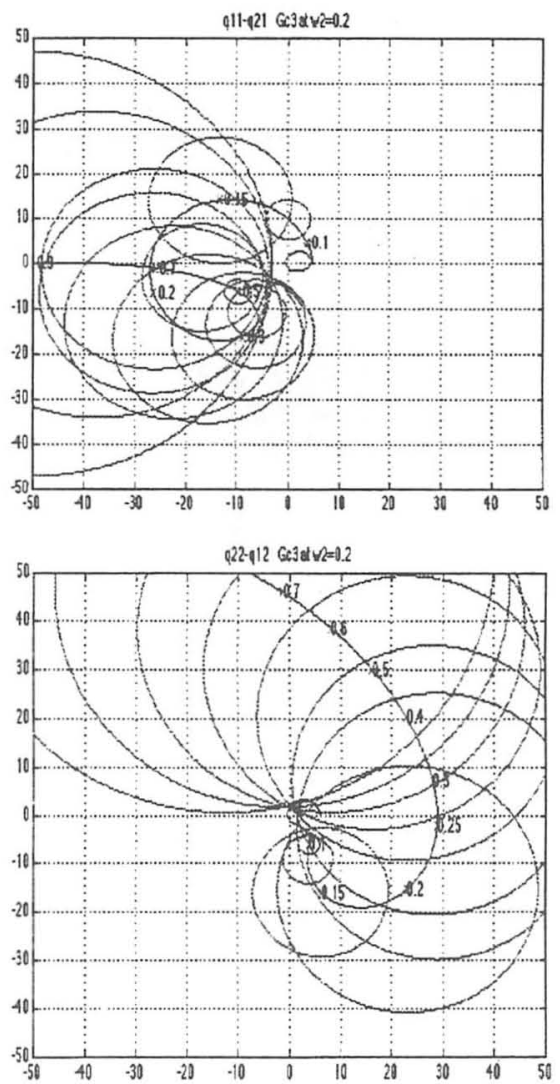

Figure 8 : Inverse Nyquist diagram by series connection

\subsubsection{Parallel connection of precompensators}

A parallel connection is also possible. Figure 9 is a block diagram of the parallel connection. The precompensator $G_{c 2}$ is designed regarding the transfer function $G_{p}(s) G_{c 1}$ as a new controlled system $G_{p}(s)^{\prime}$. Similarly the precompensator $G_{c 3}$ is designed regarding the transfer function $G_{p}(s)^{\prime} G_{c 2}$ as a new controlled system $G_{p}(s)^{\prime \prime}$. The precompensator $G_{c 1}+G_{c 2}+G_{c 3}$ connected in parallel is

$$
G_{c 1}+G_{c 2}+G_{c 3}=\left[\begin{array}{rr}
0.7407 & 2.6180 \\
-2.2479 & -0.8362
\end{array}\right]
$$

and the inverse Nyquist diagram and Gershgorin bands are shown in Fig.10, and then the diagonal dominance also realized. 


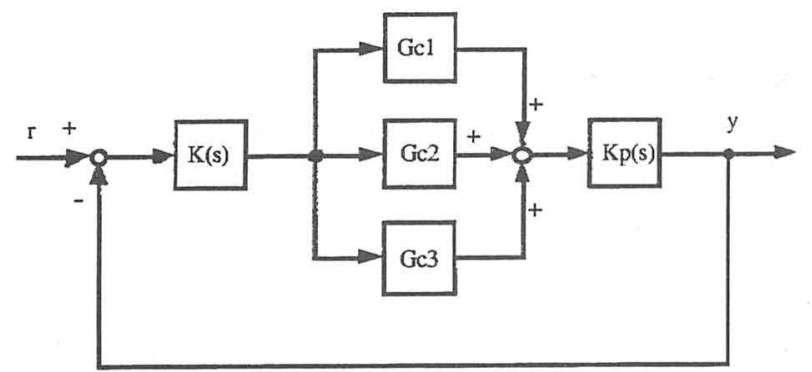

Figure 9 : Block diagram of parallel connection
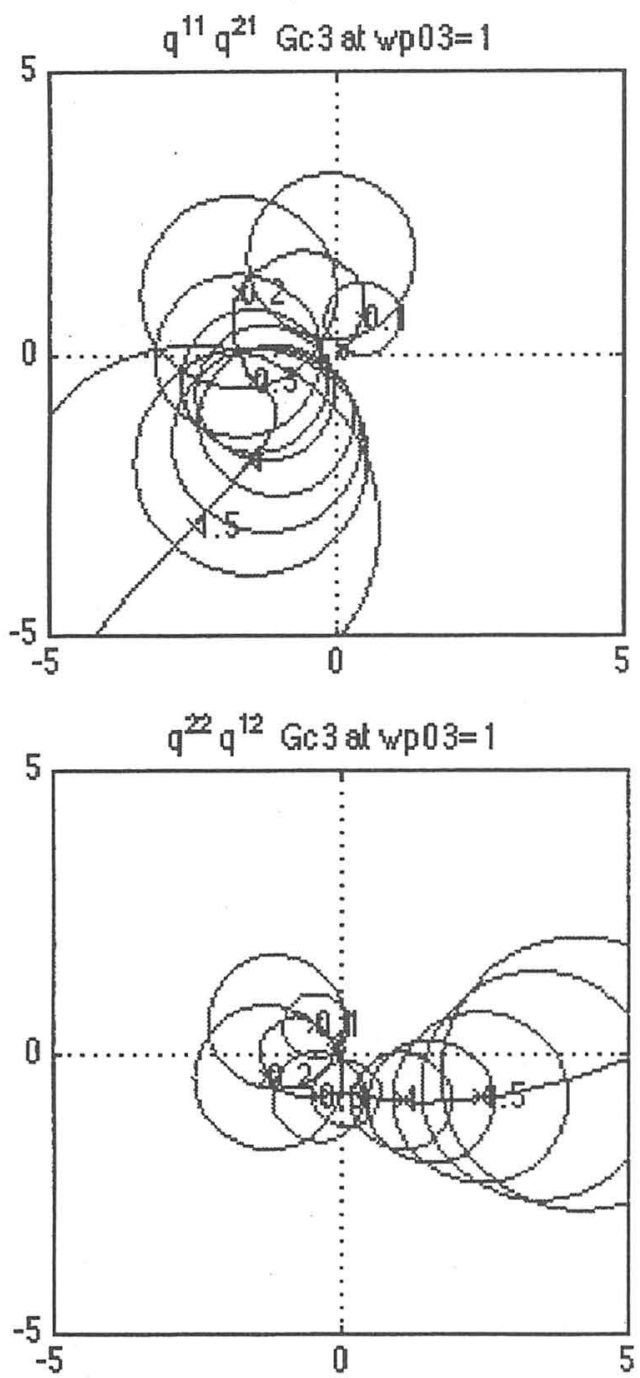

Figure 10 : Inverse Nyquist diagram by parallel connection

\subsubsection{Parallel-Series connection of precompensators}

A parallel-series connection of the precompensators is illustrated in Fig. 11 as an example. The precompensators $G_{c 11}$ and $G_{c 12}$ are designed using the hunting frequencies $\omega_{0 s}$ and $\omega_{02}$ of the result of PID control shown in Fig. 5 . If the diagonal dominance does not realize, then new precompensators $G_{c 21}$ and $G_{c 22}$ are connected. The transfer function is as

$$
\left(G_{c 11}+G_{c 12}\right)\left(G_{c 21}+G_{c 22}\right)=\left[\begin{array}{rr}
1.4073 & 2.0880 \\
-2.0346 & -1.4258
\end{array}\right]
$$

and the inverse Nyquist diagram and Gershgorin bands are shown in Fig. 12, then the diagonal dominance succeeds.

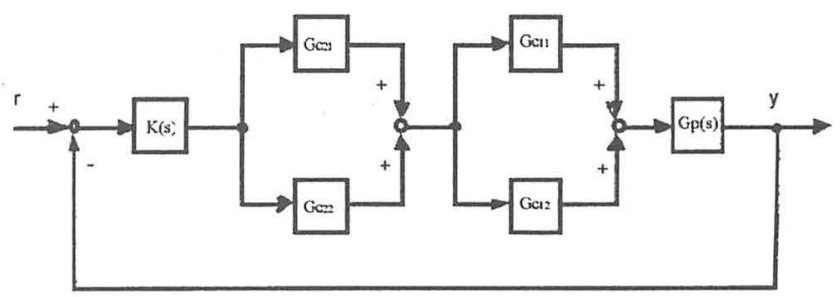

Figure 11 : Block diagram of parallel-Series connection
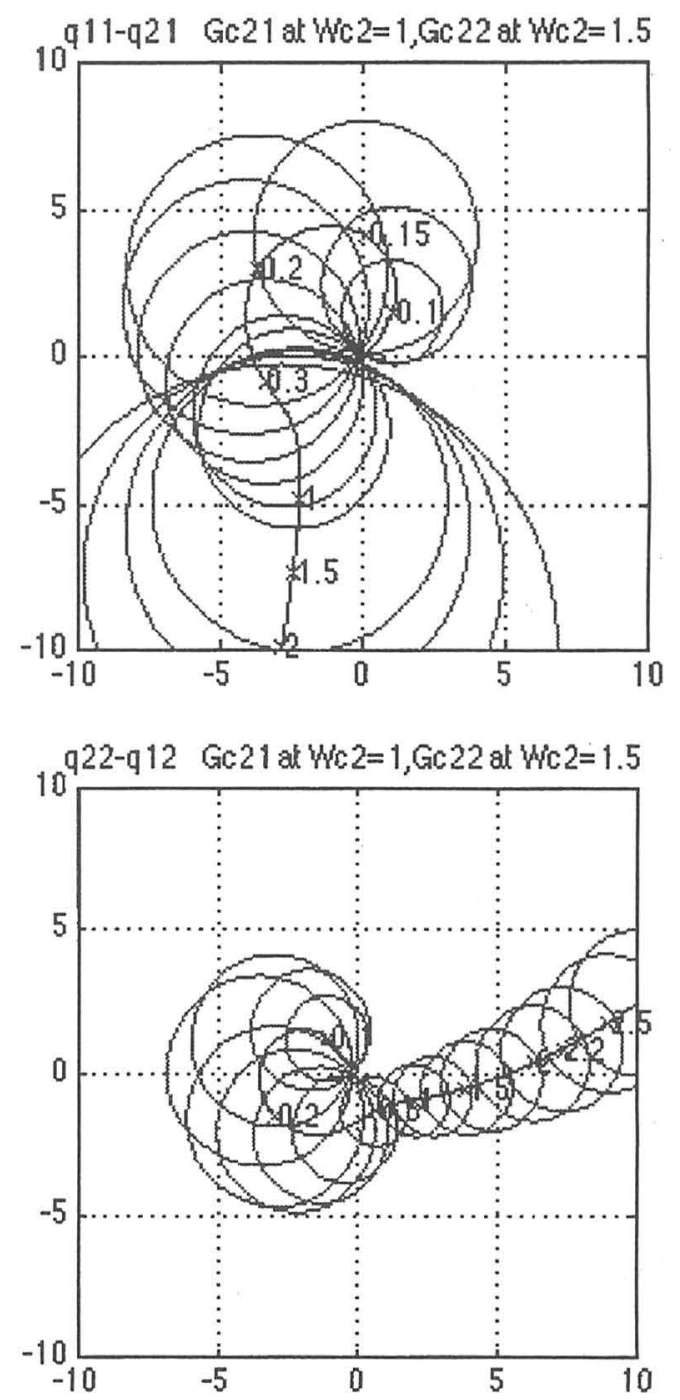

Figure 12 : Inverse Nyquist diagram by parallel-series connection

\subsection{Control experiment}

We apply the proposed precompensators with series and/or 
parallel connection to noninteracting PID control of the fluid temperature and liquid level experimental plant shown in Fig.3. The precompensators are connected to the third stage in a series described in section 4.3.2, and the PI controller is designed for each loop of the fluid temperature and liquid level controlled variables, respectively. The experimental result is shown in Fig. 13. The fluid temperature and liquid level controlled variables reach quickly the set points of the fluid temperature 50 [deg] and the liquid level 40 [cm], respectively, and furthermore the hunting does not occur in two controlled variable. As the result we can confirm the effectiveness of the proposed method.
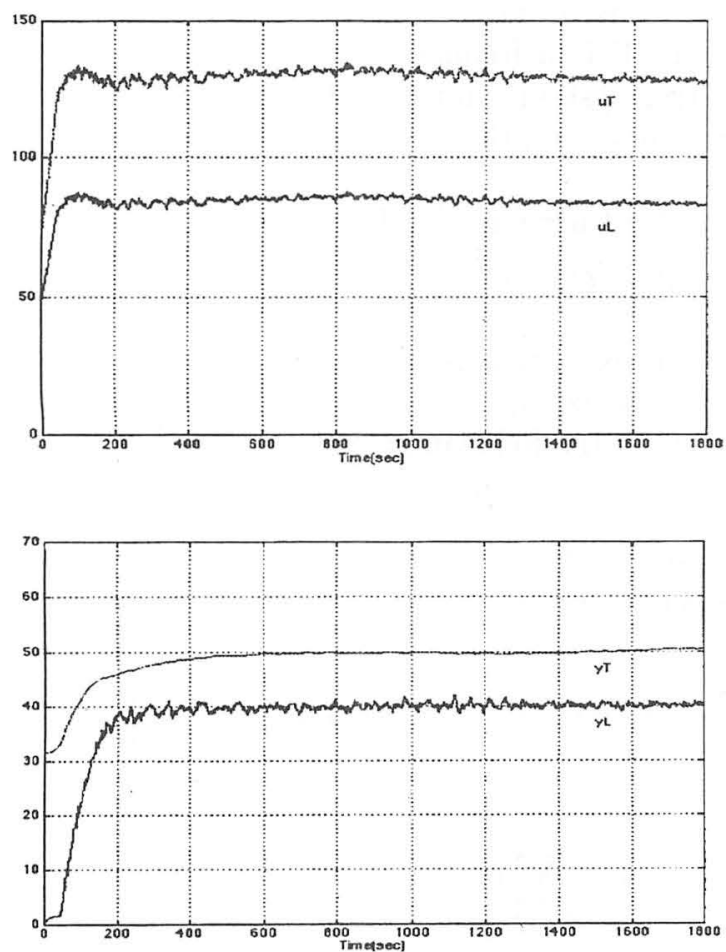

Figure 13 : Noninteracting PI control by series connection of precompensators

\section{Conclusion}

Controlled variables in multivariable systems interact each other in general, therefore, it is necessary to remove their interaction sufficiently for the design of control systems. As one of the countermeasure, the precompensator by the inverse Nyquist array method was used for noninteraction of the controlled variables. However it was difficult to remove the interaction of the controlled system, which has very strong interaction, when the precompensator is used only one. Accordingly, the series and/or parallel connections of the precompensators for noninteracting PID control were proposed in this paper, and the precompensators were applied to the control of the fluid temperature and liquid level interacting plant. As the result we could confirm the effectiveness of the proposed method.

\section{References}

[1] H.H.Rosenbrock: Design of Multivariable Control Systems using the Inverse Nyquist Array, IEE Proc., 115

[2] P.L.Falb and W.A.Wolovich: Decoupling in the Design and Synthesis of Multivariable Control Systems, IEEE, AC-12 (1967)

[3] D.J.Hawkins: Pseudo Diagonalization and the Inverse Nyquist Arroy Method, IEE Proc., 119 (1972)

[4] H.Kimura: CAD of the Multivariable Control System, J. of Soc. of Inst. and Conrl. Eng., Vol.15, No.12 (1976)

[5] M.Ito et al:: Theory of Design for Linear Control Sistems, SICE (1978)

[6] H.Lee et al.: Noninteracting PID Control of Fluid Temperature and Liquid Level Interacting System, T.IEE Japan, Vol.119-C, No.8/9 (1999) 